\section{Avaliação da implantação de programa voltado para melhoria da acessibilidade e humanização do acolhimento aos usuários na rede básica. Salvador, 2005-2008}

\section{Evaluation of the implementation of a program aiming to improve accessibility and humanization for primary health care users. Salvador, Brazil, 2005-2008}

Ligia Maria Vieira-da-Silva 1 Monique Azevedo Esperidião 2 Solange Veloso Viana 3 Vânia Sampaio Alves 4 Denise Vieira da Silva Lemos 5 Maria Constantina Caputo 6 Marcelle de Oliveira Cardoso 7 Sonia Cristina Lima Chaves 8 Luis Eugênio Portela Fernandes de Souza 9 Edyara de Moraes Santana 10 Maíra Cláudia Barbosa Araújo de Oliveira 11 Alcione Brasileiro Oliveira Cunha 12

\section{Abstract}

Objectives: to evaluate and monitor the implementation of a project aiming to broaden access and humanization of user embracement for users of the primary health care in Salvador, Brazil, between November 2005 and May 2008.

Methods: the implementation of the project was evaluated at three points in time, both externally, by a team of consultants, and internally as part of a selfevaluation by Unit Managers. An intervention logic model was designed for this purpose and a set of evaluation criteria derived from this.

Results: at the end of the first year, the implementation of program components varied from $45 \%$ to $82 \%$ of units. Six months later, implementation was more extensive. In the third and last year, in 2008, an evaluation carried out in a sample of 24 units revealed continued implementation of the project, albeit with a considerable setbacks.

Conclusions: the high degree of implementation observed, with a reduction in the size of avoidable waiting queues and improvement in access to health units in the first year, may be related to the implementation strategy adopted and to some of the specific features of municipal administration. The authors also discuss the possible reasons for the obstacles encountered within the municipal administration of the Brazilian National Health System.

Key words User embracement, Humanization of assistance, Primary health care, Monitoring

\section{Resumo}

Objetivos: avaliar e monitorar a implantação de um projeto voltado para a ampliação do acesso e a humanização do acolhimento aos usuários da rede básica de Salvador, entre novembro de 2005 e maio de 2008.

Métodos: foi realizada uma avaliação da implantação do projeto em três momentos, tanto de uma perspectiva externa, por equipe de consultores, quanto resultado de uma autoavaliação por parte dos Gerentes de Unidades. Com esse objetivo, foi elaborado um modelo lógico da intervenção, do qual foi derivada uma matriz com critérios para a avaliação.

Resultados: ao final do primeiro ano, a implantação dos componentes do programa variou entre $45 \%$ e $82 \%$ das unidades. Seis meses após, verificouse uma ampliação da implantação. No terceiro e último ano, em 2008, avaliação feita em amostra de 24 Unidades revelou persistência da implantação do projeto, porém com importantes retrocessos.

Conclusões: o elevado grau de implantação verificado, com a redução das filas evitáveis e melhoria da acessibilidade às unidades de saúde, no primeiro ano, pode estar relacionado à estratégia de implantação, bem como a algumas características da gestão municipal. Os autores discutem ainda as possiveis razões dos obstáculos encontrados no contexto da gestão municipal do Sistema Único de Saúde.

Palavras-chave Acolhimento, Humanização, Atenção básica, Monitoramento 


\section{Introdução}

Os problemas de acesso aos serviços de saúde na atenção básica, e, especialmente a existência de filas para marcação e atendimento, bem como estratégias para sua superação, têm sido descritos pela literatura internacional. A marcação de consultas para o mesmo dia em que o paciente buscou o cuidado (acesso aberto ou avançado) tem sido relatada como intervenção importante para reduzir o agendamento do paciente em longo prazo, diminuindo o tempo de espera para a consulta do médico. A implantação deste sistema requer, contudo, equilíbrio entre a oferta e a demanda, o desenvolvimento de planos de contingência para circunstâncias incomuns, além de sua adequação às práticas da atenção primária. ${ }^{1-4}$

No Brasil, o acesso aberto tem existido não como estratégia para facilitar a utilização dos serviços pelos cidadãos, mas, como decorrência da ausência de medidas específicas para organização do acolhimento aos usuários. Embora a universalização da atenção à saúde esteja constitucionalmente assegurada e a expansão do Programa de Saúde da Família (PSF) tenha em muito contribuído para esta finalidade, as barreiras organizacionais ao acesso aos serviços da rede básica, principalmente em capitais, é ainda um problema central para a consolidação do Sistema Único de Saúde (SUS) no país. 5,6

Historicamente, problemas na qualidade do cuidado à saúde, principalmente no que toca à sua dimensão interpessoal, têm gerado demandas por uma "humanização" do mesmo. Esse discurso pode ser identificado desde o início do século XX, quer nas políticas de controle do câncer na França, 7 quer no movimento da medicina preventiva. 8 No entanto, a sua tradução em medidas específicas, no Brasil, só vai ocorrer a partir da década de 1980 com o movimento da reforma sanitária.

Já como medida específica, a elaboração da Política Nacional de Humanização data de 2000. ${ }^{9}$ São diversas as concepções acerca do significado de "humanização do cuidado" que implicam em variadas práticas correspondentes. A ideia de humanização tem sido relacionada a uma série de valores vagos e incertos, como dignidade humana, ${ }^{10,11}$ remetendo à definição genérica do humanismo clássico, ${ }^{11}$ ou valores do senso comum relacionados à bondade ou altruísmo.12 Outras noções de humanização remetem diretamente à mudança na relação entre as equipes, com ênfase nas "tecnologias relacionais" como escuta, acolhimento, diálogo e negociação. ${ }^{12,13}$

A existência de filas evitáveis para a marcação de consultas e/ou utilização de serviços da rede básica pública de atenção à saúde, que resultam em sofrimento humano desnecessário, tem sido considerada como problema e obstáculo ao acesso ao cuidado, e, por si só, como componente da sua má qualidade. Esse problema, entretanto, não é recente na história das políticas públicas de saúde no Brasil. As intervenções voltadas para sua superação têm sido descritas desde os primórdios da reforma sanitária nas experiências de descentralização durante o período das Ações Integradas de Saúde (AIS) e dos Sistemas Unificados e Descentralizados de Saúde (SUDS), ${ }^{14}$ aparecendo, também, como um dos aspectos que corresponderiam à "ausência de humanidade" na organização dos serviços.

Na década de 1990, a persistência desse e de outros problemas na organização dos serviços de saúde repercutiu no acesso e na qualidade da assistência. Naquele período, a existência de proposições de modelos de atenção alternativos, referenciadas em conceitos como: vigilância da saúde, 15,16 ações programáticas, 17 vínculo e acolhimento, 18 inspirou o desenvolvimento de experiências concretas, voltadas para a melhoria da acessibilidade e do acolhimento, das quais existem alguns relatos publicados. Em São Paulo, foi operacionalizada a reorganização do sistema de acolhimento, 18 em Vitória da Conquista, entre outras medidas, foram adotados mecanismos e fluxos para o encaminhamento dos usuários no sistema de serviços $19 \mathrm{e}$ em Betim, a constituição de uma "equipe de acolhimento", resultou na melhoria da recepção aos pacientes e no aumento da oferta dos serviços de saúde. 20 Contudo, nesses trabalhos, o problema do agendamento das consultas e da extinção das filas evitáveis tem sido tematizado tangencialmente ou não tem sido objeto explícito das intervenções avaliadas. Além disso, poucos são os estudos que analisam estratégias voltadas para a implantação de programas, articuladas com o monitoramento dos progressos da sua operacionalização.

O presente artigo pretende preencher esta lacuna, apresentando os resultados do monitoramento de uma intervenção voltada tanto para a extinção de filas evitáveis, como para a humanização do acolhimento aos usuários da rede básica de saúde de Salvador, Bahia. Essa intervenção teve ainda como peculiaridade a articulação entre medidas voltadas para a reorganização do processo de trabalho nas unidades básicas com estratégias de sensibilização dos profissionais, apoiadas na técnica dos grupos operativos de Pichon- Rivière, 21 num trabalho interdisciplinar que envolveu especialistas em saúde coletiva e psicólogos sociais. 


\section{A análise da situação inicial}

Segundo dados da Assessoria Técnica da Secretaria Municipal de Saúde de Salvador, em 2004, o município dispunha de uma rede própria de serviços constituída por 107 unidades de atendimento, entre estas, 59 unidades tradicionais de atenção básica e 24 unidades de saúde da família. ${ }^{22}$ Naquele período, o município contava com 81 equipes de saúde da família implantadas, o que correspondia à cobertura populacional de 10,9\%. Em 2005, foi feita ampliação da oferta através da implantação de mais 29 equipes de saúde da família ${ }^{23}$ e da criação de nove unidades de saúde da família, o que aumentou a cobertura do Programa de Saúde da Família para $14,8 \%$. A despeito dessas medidas, o acesso e o acolhimento aos usuários na rede básica apresentavam diversos problemas. Em praticamente todas as unidades havia filas para o atendimento em decorrência tanto da existência de desequilíbrio entre oferta e demanda como em consequência de sistemas restritivos de marcação de consulta (semanal, mensal ou periódico).

A situação do acesso à rede básica de saúde de Salvador foi analisada, em setembro de 2005, a partir de informações oriundas de um inquérito realizado antes da implantação do projeto para a melhoria da acessibilidade e humanização do acolhimento. A amostra desse primeiro inquérito não foi probabilística, tendo em vista que o mesmo foi desenvolvido como atividade prática de 86 alunos de cursos da área de saúde da Universidade Federal da Bahia (UFBA) que cursavam, em 2005, a disciplina Políticas de Saúde do Instituto de Saúde Coletiva da Universidade Federal da Bahia. Foram entrevistados 59 gerentes ou integrantes de comissão gerencial de unidades de 11 entre os 12 distritos ( $46 \%$ do total de 127) e 403 usuários de distritos sanitários de Salvador. Foram entrevistados os usuários que se encontravam na sala de espera, selecionados de forma intencional entre mulheres e homens acima de 18 anos, de maneira a estarem representados acompanhantes de crianças, mulheres adultas, homens adultos e idosos. Também foram entrevistados os profissionais que se encontravam trabalhando no momento e disponíveis para a entrevista, portanto, selecionados por conveniência (médico, enfermeiro e odontólogo). Para abordagem dos entrevistados, foram observados os aspectos éticos previstos da Resolução CNS 196/96 e suas complementares, como utilização do termo de consentimento livre e esclarecido. Contudo, como a perda de informações foi superior a $50 \%$ em diversas questões, esse material não pôde ser aproveitado na sua totalidade para fins de comparação com as avaliações subsequentes, todavia, forneceu subsídios para as decisões referentes à implantação e permitiu fazer algumas inferências sobre a situação anterior à intervenção.

$\mathrm{O}$ inquérito realizado pelos estudantes de medicina confirmou as impressões iniciais relativas ao acesso aos serviços da rede básica de Salvador: não havia uma política municipal para a melhoria do acesso, sendo que cada unidade organizava a recepção aos pacientes de forma autônoma e desarticulada. Segundo informação dos gerentes, o agendamento de consultas era feito predominantemente de forma irregular: diariamente, porém em apenas um turno (22\% das unidades), semanal (34\%), mensal ou quinzenal (6\%). Em apenas $14(24 \%)$ das 59 unidades visitadas, a marcação era permanente (diária e nos dois turnos). Em 13 (22\%) das unidades, ainda havia o sistema de distribuição de senhas e atendimento por ordem de chegada. As filas existiam em $30(51 \%)$ das unidades visitadas, devido, segundo opinião dos gerentes, principalmente ao desequilíbrio entre oferta e demanda (31\%) e problemas na estrutura das unidades (12\%), mas também devido à "cultura" dos usuários em "chegar cedo" (5\%). Em diversas unidades, essas filas se iniciavam na madrugada. Em uma Unidade, a fila se iniciava no dia anterior, tendo sido motivo de noticiário de jornais locais. 24 Além das filas, as dificuldades referidas pelos usuários entrevistados para a marcação de consultas, eram: a) problemas concernentes à longa espera pelo dia de marcação de consulta; b) ausência ou número limitado de vagas; c) o longo período de espera entre a marcação e a realização da consulta; d) a inadequação do espaço físico, ausência de assentos para os pacientes, o que impunha a espera em posição desconfortável e inadequada; e) dificuldades na relação entre profissionais e usuários (descaso, desatenção, desrespeito); f) limitação dos dias para a marcação de consulta; g) escassez ou falta de informação sobre os dias de marcação de consulta.

A intervenção - o projeto para a melhoria da acessibilidade e humanização do acolhimento na rede básica de Salvador

Em Julho de 2005 foi formulado o projeto para a melhoria da acessibilidade na rede básica de Salvador, 25 com os seguintes objetivos: melhoria da acessibilidade da população à rede básica; implantação de sistema de marcação de consultas; extinção de filas evitáveis; promoção de maior integração das equipes das unidades básicas de saúde, aumentando o envolvimento delas com o projeto. Os princípios 
norteadores foram: não rechaço à demanda; escuta sensível e qualificada; redefinição do fluxo de atendimento na rede de serviços de saúde. As atividades realizadas para atingir os objetivos incluíram, em uma primeira etapa: a implantação de sistema de marcação permanente de consultas (a marcação de consultas deveria ser feita de forma presencial ou por telefone, em todos os turnos da semana, para todas as especialidades); a implantação de lista de espera voltada para viabilizar atendimentos no mesmo dia da procura, e, ao mesmo tempo reduzir o absenteísmo nos casos de desistência ou não comparecimento do usuário agendado. Ao mesmo tempo, visando garantir o atendimento no mesmo dia da procura para situações classificadas como prioritárias, deveria ser instituída uma reserva estratégica de consultas que corresponderia a um número de vagas que não seriam agendadas previamente. Numa segunda etapa incluíram-se: a designação de profissional de nível médio para a recepção ao usuário; a criação de comissão de acolhimento e classificação de prioridades e riscos, que deveria ser composta por profissionais de nível superior, responsáveis pela avaliação de risco, com protocolo pré-estabelecido, objetivando selecionar as prioridades para o preenchimento da reserva estratégica de vagas, apoiadas em critérios técnicos; a designação de gestores de caso (deveria ser designado profissional de nível superior para apoiar grupos específicos de usuários, como idosos, portadores de necessidades especiais e usuários sem uma rede social de apoio); a organização da oferta - que corresponderia, numa segunda etapa do projeto, à implementação das ações programáticas para problemas priorizados pelas unidades na perspectiva da vigilância à saúde. Por último o projeto incluía a proposição de criação de grupos de trabalho em humanização constituídos pelo gestor e por outros profissionais (médicos, enfermeiros, assistentes sociais, recepcionistas), com o objetivo de coordenar a implantação do projeto nos diversos níveis da rede.

As estratégias de implantação envolveram inicialmente a criação de consenso entre coordenadores de distritos e gerentes de unidades acerca dos objetivos e estratégias do projeto. Com essa finalidade, foram utilizadas técnicas de consenso do tipo grupo nominal. ${ }^{25} \mathrm{Em}$ seguida, foi realizado um seminário cujo objetivo era mostrar a viabilidade do projeto para os profissionais, por meio da discussão de experiências similares, consideradas como bem sucedidas, desenvolvidas em outros Estados da Federação. Posteriormente, foram realizadas 23 oficinas de sensibilização envolvendo 1725 profissionais e 183 gerentes de unidades de saúde dos 12 distritos sanitários. Além disso, foram realizadas quatro oficinas para a capacitação dos servidores da recepção e portaria, envolvendo cerca de 150 pessoas. O desenho das oficinas contemplou quatro momentos: o primeiro era dedicado ao levantamento de expectativas e debate sobre as condições de trabalho. No segundo momento, era abordada a convivência em grupo, as relações interpessoais dentro das equipes de trabalho, assim como o processo de comunicação. $\mathrm{O}$ terceiro momento era dedicado à análise da percepção dos servidores das suas práticas atuais, através da dramatização de cenas cotidianas. No quarto momento da oficina, os grupos delineavam as medidas preliminares de mudança viáveis dentro de cada unidade. Do ponto de vista metodológico, foi utilizado o marco teórico da psicologia social de Pichon-Rivière 21 e a técnica dos grupos operativos integrada a recursos artísticos do cinema, teatro e música.

Para subsidiar o processo de implantação foi elaborado manual para orientação aos profissionais e gerentes. 26 Outra estratégia da implantação foi a constituição de rede de grupos de trabalho de humanização no nível central, nos distritos sanitários e em grande parte das unidades. Com o objetivo de propiciar um espaço para o intercâmbio de experiências de implantação entre as unidades foi realizado um seminário onde foram apresentadas 13 das 24 experiências consideradas como mais avançadas. Ao final do processo de implantação, o projeto, inicialmente denominado "Projeto para melhoria da acessibilidade", tendo seus objetivos consistentes com a política de humanização do Ministério da Saúde incorporou o termo "humanização", que passou a predominar, sendo, ao final do período, conhecido como o "Humaniza SUS" da Secretaria Municipal de Saúde de Salvador. ${ }^{26}$

\section{Métodos}

Após a realização das oficinas de sensibilização, uma equipe técnica acompanhou e apoiou a implantação do projeto nas Unidades de Saúde. Foram feitas avaliações em três momentos: após cinco meses de implantação (Tabela 1), após 16 meses (Tabelas 2 e 3) e ao final, após 28 meses. Esse acompanhamento correspondeu a um monitoramento da intervenção. A avaliação foi aqui considerada como um julgamento que se faz sobre as práticas, que pode ser pontual ou corresponder a um acompanhamento sistemático; nesse último caso pode ser denominado de monitoramento. 27,28

Além disso, ao final do primeiro ano, foi realizada pesquisa avaliativa, através de inquérito, em 


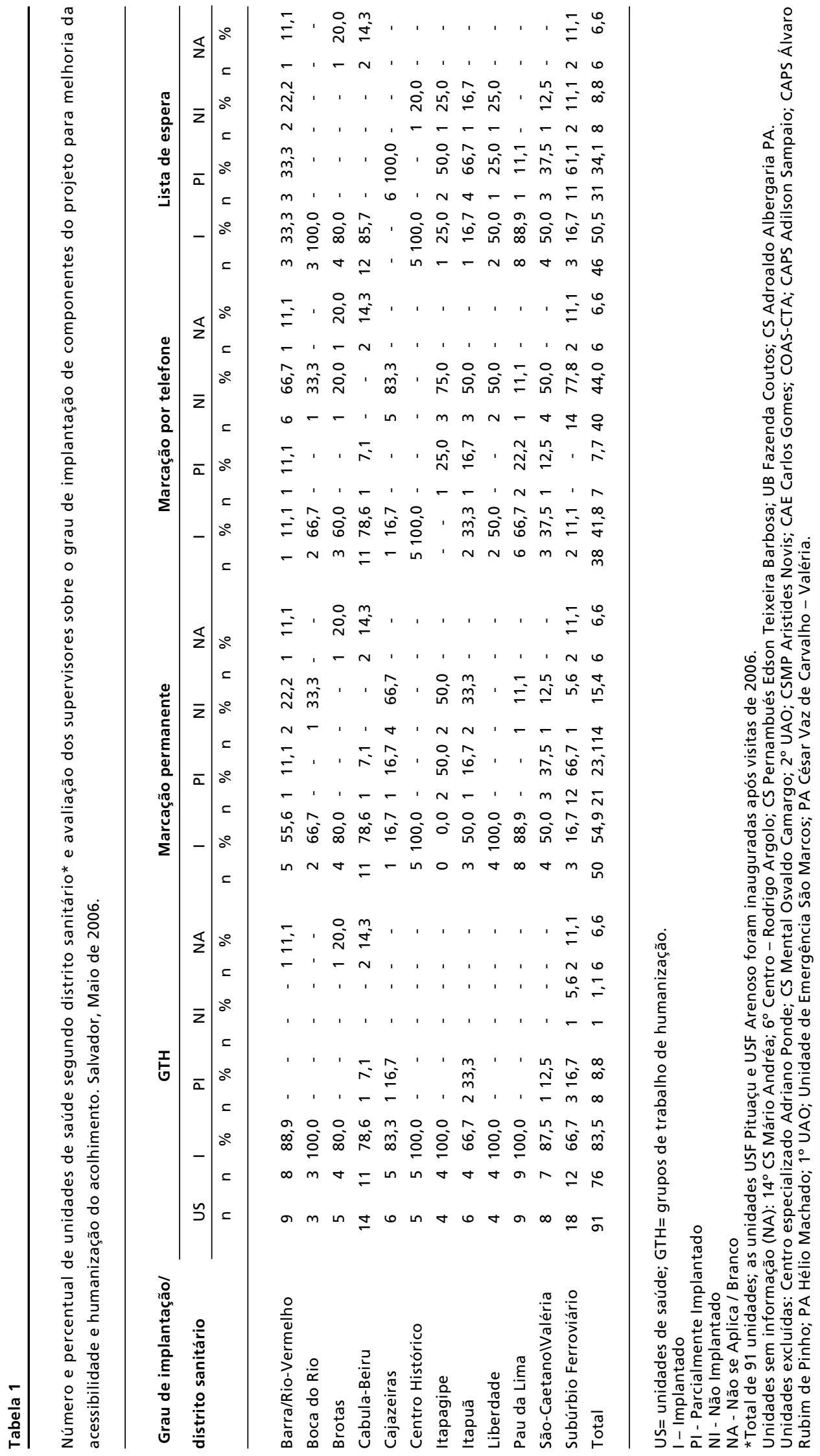




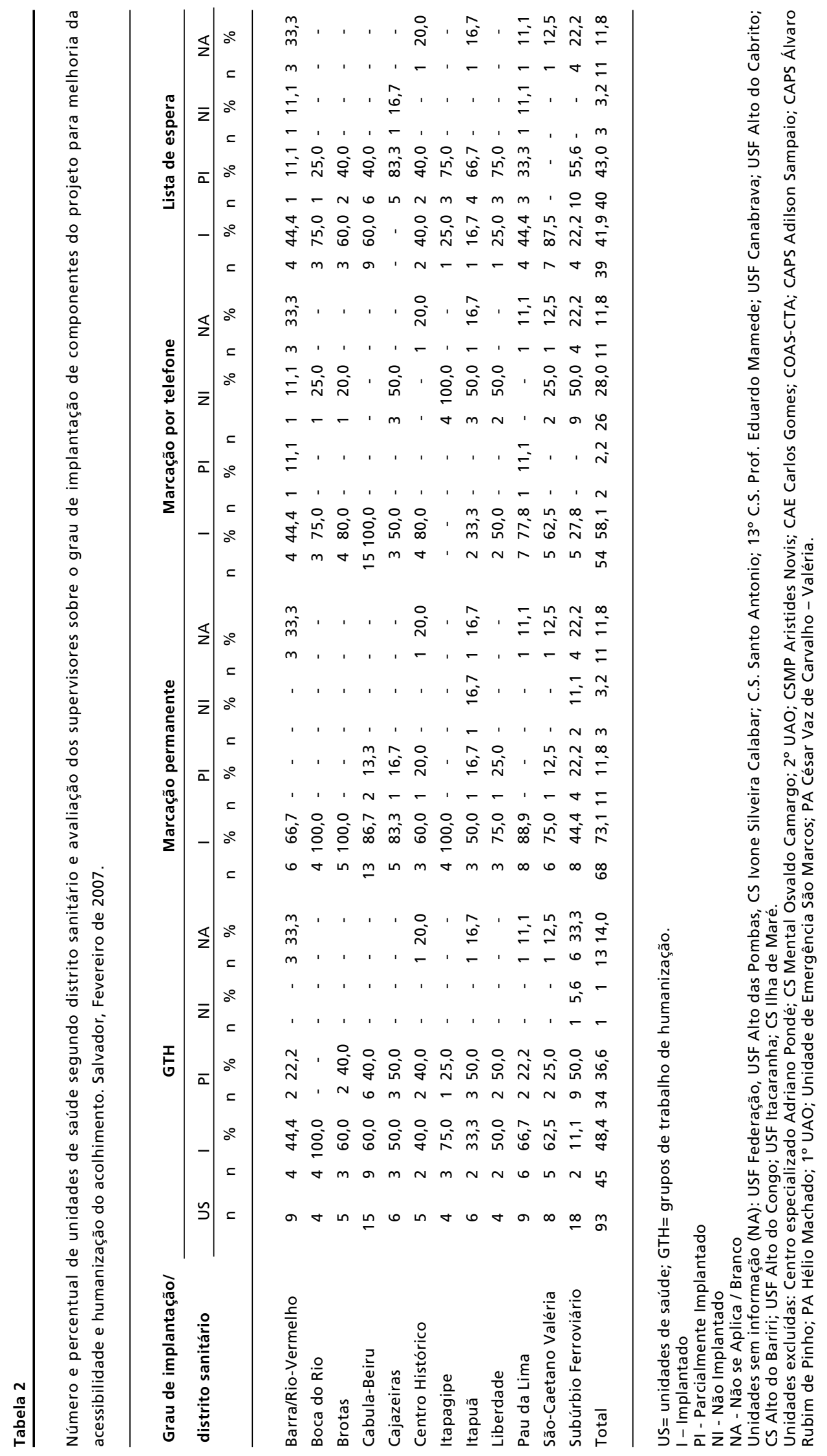




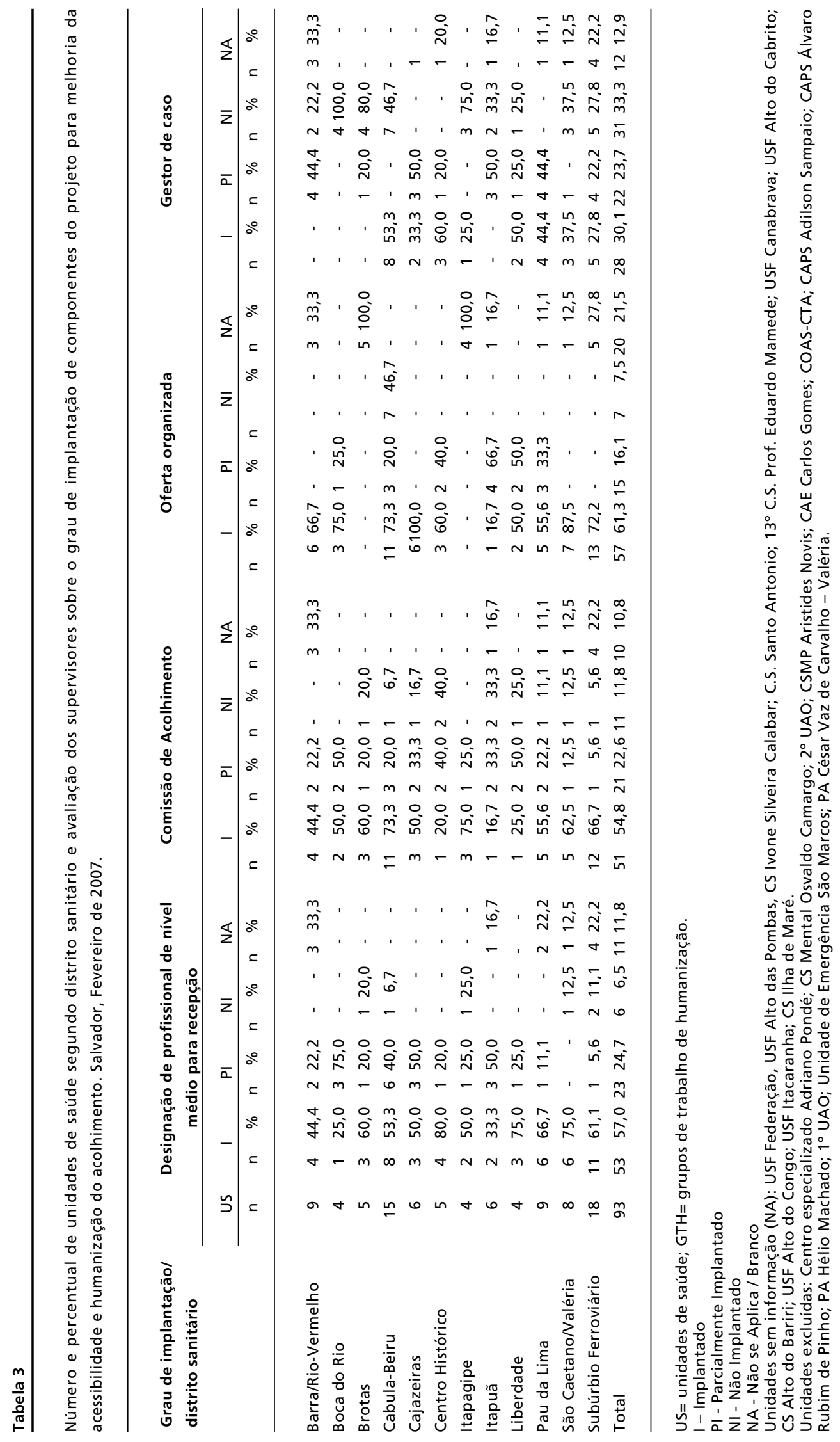


amostra probabilística de unidades cujos resultados foram objeto de outra publicação. ${ }^{29}$ Foram comparados os resultados obtidos nas unidades onde o programa estava implantado com aqueles observados onde não havia implantação do mesmo. ${ }^{29}$

Para o monitoramento foi elaborado o modelo lógico da intervenção envolvendo os objetivos, atividades e resultados esperados (Tabela 4), que orientou o desenvolvimento subsequente de matriz de critérios (Tabela 5), também utilizada em projeto de pesquisa, desenvolvido após o final do primeiro ano, e que avaliou a efetividade da intervenção. ${ }^{29} \mathrm{~A}$ referida matriz foi adaptada e utilizada para a avaliação, tanto de uma perspectiva externa, pelos consul- tores, como de uma perspectiva interna, pelos gerentes das unidades, numa autoavaliação, de forma sistemática em todas as unidades da rede básica nos dois primeiros momentos. ${ }^{26}$ Apenas no terceiro momento do monitoramento, em fevereiro de 2008, é que o julgamento foi feito exclusivamente pela equipe técnica de consultores, responsável pelo acompanhamento do programa, em amostra composta por duas unidades sorteadas aleatoriamente de cada distrito. Essa amostra foi a mesma utilizada em pesquisa avaliativa sobre a efetividade do programa e seu cálculo encontra-se detalhado em outra publicação. 29

Tabela 4

Modelo lógico do projeto para a melhoria da acessibilidade e humanização do acolhimento na rede básica do Sistema Único de Saúde. Salvador, Bahia.

\begin{tabular}{|c|c|c|}
\hline Objetivos & Atividades & Resultados \\
\hline $\begin{array}{l}\text { Extinguir as filas } \\
\text { evitáveis }\end{array}$ & $\begin{array}{l}\text { - Implantação de sistema permanente de } \\
\text { marcação de consultas; } \\
\text { - Marcação de consulta por telefone; } \\
\text { - Criação de lista de espera para as consultas } \\
\text { eletivas para auxiliar no processo de reedefinição } \\
\text { do fluxo }\end{array}$ & $\begin{array}{l}\text { - Extinção das filas evitáveis } \\
\text { - Diminuição do tempo de espera } \\
\text { na fila; } \\
\text { - Diminuição no tempo entre } \\
\text { agendamento e realização da } \\
\text { consulta }\end{array}$ \\
\hline $\begin{array}{l}\text { Melhoria do } \\
\text { acolhimento ao } \\
\text { usuário na unidade }\end{array}$ & $\begin{array}{l}\text { - Criação de comissão de colhimento composta } \\
\text { por médicos e/ou enfermeiros; } \\
\text { - Definição de situações que devem ser } \\
\text { consideradas de pronto atendimento e eletivas; } \\
\text { - Designação de profissional de nível médio para } \\
\text { receber todo o paciente que entra na unidade e } \\
\text { orientá-lo quanto à utilização dos serviços }\end{array}$ & $\begin{array}{l}\text { - Diminuição das consultas } \\
\text { consideradas como desnecessárias; } \\
\text { - Aumento da satisfação dos } \\
\text { usuários }\end{array}$ \\
\hline $\begin{array}{l}\text { Promover maior } \\
\text { integração das } \\
\text { equipes das unidades } \\
\text { básicas de saúde, } \\
\text { aumentando o } \\
\text { envolvimento dessas } \\
\text { com o projeto }\end{array}$ & $\begin{array}{l}\text { - Realização de oficinas de sensibi-lização com } \\
\text { profissionais das unidades; } \\
\text { - Realização de seminário sobre as experiências } \\
\text { exitosas de outros municí-pios do país }\end{array}$ & $\begin{array}{l}\text { - Aumento da satisfação dos } \\
\text { profissionais; } \\
\text { - Maior envolvimento dos } \\
\text { profissionais com o Sistema Único } \\
\text { de Saúde }\end{array}$ \\
\hline
\end{tabular}


Matriz de critérios para a apreciação do grau de implantação e monitoramento do projeto para a melhoria da acessibilidade e humanização do acolhimento na rede básica do Sistema Único de Saúde. Salvador, Bahia, 2005.

\begin{tabular}{|c|c|c|c|}
\hline \multirow[t]{2}{*}{ Ação/atividade } & \multicolumn{3}{|c|}{ Classificação do grau de implantação (pontuação máxima) } \\
\hline & Não implantado & Parcialmente implantado & Implantado \\
\hline $\begin{array}{l}\text { Implantação de } \\
\text { sistema permanente } \\
\text { de marcação de } \\
\text { consultas }\end{array}$ & $\begin{array}{l}\text { A marcação de consultas } \\
\text { é feita semanalmente }\end{array}$ & $\begin{array}{l}\text { Há marcação diária em apenas um } \\
\text { turno }\end{array}$ & $\begin{array}{l}\text { Há marcação diária, em } \\
\text { qualquer horário } \\
\text { administrativo }\end{array}$ \\
\hline $\begin{array}{l}\text { Marcação de } \\
\text { consulta por } \\
\text { telefone }\end{array}$ & $\begin{array}{l}\text { A marcação de consulta } \\
\text { por telefone não é feita }\end{array}$ & $\begin{array}{l}\text { Há marcação para algumas } \\
\text { especialidades ou programas }\end{array}$ & $\begin{array}{l}\text { A marcação por telefone } \\
\text { é feita para todos os } \\
\text { programas e serviços }\end{array}$ \\
\hline $\begin{array}{l}\text { Criação de lista de } \\
\text { espera }\end{array}$ & $\begin{array}{l}\text { Os pacientes que faltam } \\
\text { não são substituídos }\end{array}$ & $\begin{array}{l}\text { Há uma substituição informal das } \\
\text { faltas }\end{array}$ & $\begin{array}{l}\text { A lista de espera é usa- } \\
\text { da, otimizando o atendi- } \\
\text { mento }\end{array}$ \\
\hline $\begin{array}{l}\text { Organização do } \\
\text { grupo de trabalho } \\
\text { de humanização na } \\
\text { Unidade }\end{array}$ & $\begin{array}{l}\text { Profissionais } \\
\text { mobilizados com a } \\
\text { proposta; não foi } \\
\text { realizada reunião para } \\
\text { discussão e elaboração do } \\
\text { plano (0) }\end{array}$ & $\begin{array}{l}\text { Mínimo de dois a três profissionais } \\
\text { mobilizados, realização de pelo } \\
\text { menos uma reunião para discussão } \\
\text { e elaboração da matriz do plano; } \\
\text { gerente não participa do GTH }\end{array}$ & $\begin{array}{l}\text { Mais de três profis- } \\
\text { sionais mobilizados, o } \\
\text { gerente integra o GHT, } \\
\text { realização de pelo } \\
\text { menos uma reunião } \\
\text { quinzenal para a discus- } \\
\text { são da implementação } \\
\text { do plano }\end{array}$ \\
\hline
\end{tabular}

GTH= grupos de trabalho de humanização.

\section{Resultados e Discussão}

\section{O monitoramento da implantação}

O monitoramento do processo de implantação revelou que a dinâmica criada durante os seminários de sensibilização gerou um envolvimento com o projeto de acolhimento, a despeito das difíceis condições de trabalho descritas pelos participantes. As oficinas mostraram ser possível aumentar a motivação de quem já tinha uma disponibilidade interna e resgatar parcialmente aqueles que estavam desmotivados. As oficinas de sensibilização contribuíram ainda para que as pessoas refletissem sobre as práticas profissionais e pessoais e incorporassem a importância da escuta do "outro", como é possível verificar nos depoimentos de alguns dos participantes das oficinas:

"Metodologia que contribuiu para (...), um processo de reflexão sobre minha prática profissional e minha vida pessoal (...) aprendi no mínimo a ouvir o outro..."
"Entendi que não posso desejar que o outro se torne coresponsável por sua saúde se ele não é respeitado, não é ouvido, (...). Compreender isso na minha vida pessoal foi crucial para refletir isso na minha prática profissional".

Segundo informações fornecidas pelos coordenadores de distrito, a partir da autoaplicação da matriz de monitoramento (Tabela 4), os Grupos de Trabalho de Humanização (GTH) estavam implantados em maio de 2006 em 83,5\% (76) das unidades; a marcação permanente de consultas funcionava em $55 \%$ (50) das unidades, a marcação por telefone em $41,8 \%(38)$ e a lista de espera em 50,5\% (46), como se observa na Tabela 1. Essa situação, que pode ser caracterizada como estágio de implantação intermediário, para a maioria dessas ações, exceção apenas aos GTH, que foram considerados como em estágio avançado da implantação ( $>66 \%$ das unidades), parece ter sido impulsionada pela vinculação da remuneração à operacionalização das ações de acolhimento, estabelecida pela Secretaria Municipal de Saúde através de portaria específica. 
Os demais componentes do primeiro estágio e aqueles do segundo estágio existiam de forma bastante localizada e incipiente (comissão de acolhimento, gestor de caso e oferta organizada). No seminário para avaliação das experiências exitosas, iniciativas inovadoras para além das medidas propostas pelo plano foram apresentadas por 13 unidades de saúde, selecionadas entre 24 inscritas. ${ }^{26}$

Ao final do primeiro ano, verificou-se uma variação no grau de implantação entre os diferentes distritos. A implantação do GTH ocorreu em mais de $80 \%$ das unidades de dez distritos, o que revela uma adesão inicial ao projeto. Embora a medida de maior impacto na reorganização do processo de trabalho, a saber, a marcação permanente de consultas, tenha sido implantada em $50 \%$ ou mais das unidades, na maioria dos distritos, em três casos verificou-se praticamente ausência de implantação. Em apenas quatro distritos sanitários (Centro Histórico, Cabula Beiru, Pau da Lima e Boca do Rio) houve uma implantação avançada ( $\geq 66 \%$ ) dos quatro componentes do projeto (Tabela 1).

Em 2007, um ano e meio após a implantação do projeto, o monitoramento revelou uma ampliação da implantação dos principais componentes: a marcação permanente aumentou de $54,9 \%$ para $73,1 \%$ e a marcação por telefone de $41,8 \%$ para $58,1 \%$. Essa ampliação foi observada em mais de $75 \%$ das unidades, em 8 dos 12 distritos analisados. Contudo, houve redução na proporção de unidades com GTH em funcionamento e com lista de espera. Essas modificações ocorreram de forma heterogênea nos distritos avaliados (Tabela 2). Apenas no distrito da Boca do Rio houve ampliação da implantação de todos os componentes do projeto. Chama a atenção que a lista de espera foi implantada em menos de $50 \%$ das unidades em 7 dos 12 distritos analisados (Tabela 2). Verificou-se ainda, ter havido uma ampliação de dois para cinco do número de distritos com mais de $75 \%$ das unidades onde a marcação por telefone havia sido implantada.

Ainda nesse mesmo ano, foi formulado projeto específico para a incorporação dos componentes da segunda etapa do projeto, a saber: designação de profissional de nível médio para recepção, comissão de acolhimento, oferta organizada e gestor de caso. A designação de profissionais de nível médio para recepção foi verificada em $57 \%$ (53) das unidades. A comissão de acolhimento foi implantada em 54,8\% (51) das unidades. Experiências localizadas de implantação do gestor de caso foram observadas em $30,1 \%$ (28) das unidades e ações voltadas para a organização da oferta foram registradas em $61,3 \%$ (57) das unidades (Tabela 3). Apenas nos distritos do
Centro Histórico, Liberdade e São Caetano/Valéria a implantação do profissional de nível médio ocorreu em mais de $75 \%$ das unidades. Já a comissão de acolhimento encontrava-se implantada em mais de $70 \%$ das unidades apenas nos distritos Cabula-Beiru e Itapagipe (Tabela 3). Embora a oferta organizada tenha sido referida como implantada em mais de $70 \%$ de cinco distritos, a concepção do que seria a oferta organizada variou muito, dificultando a interpretação deste resultado (Tabela 3). Entre os novos componentes, o menos implantado foi o gestor de caso: em apenas três distritos houve implantação em mais de $50 \%$ das unidades.

A variabilidade na implantação dos diversos componentes do projeto, entre os distritos sanitários, está relacionada principalmente à diversidade das características das equipes gestoras dos distritos e unidades, a mudanças no quadro dirigente da Secretaria Municipal de Saúde (SMS) bem como a problemas relacionados à gestão da atenção primária, incluindo dificuldades no abastecimento da rede básica e contratação de pessoal.

Tanto o monitoramento feito pela cooperação técnica do Instituto de Saúde Coletiva, quanto a autoavaliação dos coordenadores e gerentes de unidades, foi consistente com pesquisa realizada em amostra probabilística de 24 unidades e de 710 usuários entre julho e agosto de 2006, que revelou efetividade do projeto quando comparadas unidades com o projeto implantado com aquelas onde o mesmo não estava implantado. $29 \mathrm{O}$ melhor acesso à atenção básica foi verificado nas unidades onde o projeto foi considerado implantado em comparação às unidades em que o mesmo não foi implantado, sobretudo no que se refere ao horário de chegada na fila e à redução do tempo de espera para marcação de consulta. A percepção positiva sobre a mudança nos sistema de marcação e acolhimento foi maior entre usuários das unidades consideradas implantadas. 29

Em 2008, a avaliação feita em amostra de 24 unidades revelou persistência da implantação do projeto. A marcação permanente ainda foi observada em 54,2\% (13) unidades, a marcação por telefone em $50 \%$ delas (12) e a lista de espera em 12,5\% (3). Portanto, na maioria delas o projeto estava parcialmente implantado, revelando retrocesso decorrente principalmente da instabilidade do quadro de pessoal e do desabastecimento da rede. Cabe destacar que esse foi um monitoramento mantido na mesma amostra aleatória de unidades de saúde de estudo anterior. ${ }^{29}$ Além disso, avaliação qualitativa, feita em maio e junho de 2008, em 28 unidades de cinco distritos sanitários, por meio de visitas técnicas da 
equipe de consultores e técnicos da Secretaria Municipal de Saúde, revelou que o projeto persistia implantado em grande parte das mesmas. Havia diversos retrocessos, que variavam de distrito para distrito, principalmente relacionados aos problemas acima mencionados: falta de médicos, instabilidade trabalhista dos profissionais e desabastecimento da rede. 26

A extensão do projeto para a rede credenciada foi planejada e um diagnóstico da situação de acolhimento na rede privada vinculada ao SUS chegou a ser realizado. Foi elaborado pré-projeto voltado para a melhoria da acessibilidade e humanização do acolhimento para aquele subsetor que não chegou a ser implantado devido à segunda mudança de secretário municipal de saúde em um período de três anos.

\section{A gestão municipal do SUS em Salvador e os} obstáculos à implantação do projeto

Os principais obstáculos à consolidação do projeto identificados pela equipe técnica, gerentes e coordenadores foram os seguintes: a) desmotivação muito grande dos profissionais das unidades devido à falta de condições de trabalho; b) persistência de problemas no abastecimento da rede; c) escassez de pessoal qualificado, principalmente médicos clínicos e pediatras, bem como odontólogos. Em algumas unidades, o atendimento foi interrompido por esse motivo; d) problemas trabalhistas variados (atraso de salário e indefinição do tipo de vínculo trabalhista dos profissionais); e) problemas na gestão de pessoa (má distribuição dos profissionais; não cumprimento de carga horária; remuneração variável e multiplicidade de vínculos); f) espaço físico inadequado em muitas unidades; g) subutilização de cotas reguladas da rede credenciada; g) dualidade de poder entre gerentes de terceirizadas e da rede própria; $h$ ) mudança de secretários de saúde, com modificações nas equipes dirigentes. Destaca-se que o não pagamento de fornecedores e prestadores de serviços com recursos municipais, em 2006, teve por consequência a falta de insumos básicos nas unidades de saúde e paralisações, passeatas e greves organizadas pelos trabalhadores terceirizados, com salários atrasados.

Os retrocessos foram muitos para o programa de saúde da família. ${ }^{30}$ Este não só teve sua expansão contida, como também teve sua qualidade comprometida; as ações de prevenção da dengue perderam intensidade; a implantação do centro de referência da saúde do trabalhador foi reiteradamente adiada; as obras de construção de 15 novas unidades de saúde foram praticamente paralisadas. 30

\section{Considerações finais}

O projeto para melhoria da acessibilidade e humanização do acolhimento atingiu parcialmente seus objetivos centrais, a saber, a extinção das filas evitáveis e introdução de ações voltadas para a humanização do acolhimento aos usuários. Esses resultados positivos foram evidenciados através de monitoramento externo, autoavaliação e pesquisa avaliativa. A articulação dessas diversas estratégias de avaliação que mobilizaram diversas técnicas, tais como inquérito, observação, análise estatística, análise do discurso, revelou o potencial da articulação entre a pesquisa, a gestão e a docência.

As condições de possibilidade do êxito dessa intervenção, ainda que temporário, dado que a descontinuidade administrativa interrompeu a implantação do projeto, relacionam-se com diversos aspectos. Em primeiro lugar, às características do governo municipal que, embora não tivesse um projeto de governo com prioridade clara para a reorganização do setor saúde, permitiu, pela natureza da coligação política, a ocupação de cargos estratégicos por agentes vinculados ao projeto da reforma sanitária, entre os quais dois dos secretários municipais de saúde. Houve coincidência temporal com a formulação e implantação da política nacional de humanização, que legitimou e fortaleceu a intervenção. A prioridade dada pela direção da secretaria municipal ao projeto assegurou uma relativa capacidade de governo e governabilidade para o mesmo. $\mathrm{O}$ segundo componente que contribuiu para o êxito foi a boa delimitação dos objetivos da intervenção, bem como a adequação das medidas propostas, o que permitiu que a utilização da técnica de consenso do grupo nominal resultasse na ampliação da adesão dos coordenadores de distrito e gerentes. Porém, essa dimensão racional e participativa não teria sido por si só suficiente para o elevado grau de adesão se os dois outros elementos citados anteriormente não tivessem sido agregados. O terceiro componente foi a adoção de uma estratégia de implantação que ultrapassou as "capacitações" usuais. A realização de seminários de sensibilização com o apoio de consultores da área de psicologia social foi decisivo para mobilizar gerentes e profissionais. Um quarto aspecto que influiu no êxito foi a existência de uma equipe técnica de supervisão multidisciplinar, com grande participação de psicólogos, porém incluindo enfermeira, odontóloga, nutricionista e médica, todas com formação em saúde coletiva. Essa equipe foi responsável pelo acompanhamento cotidiano da implantação, pela elaboração dos instrumentos (plano, manual, roteiros e matrizes) e pelo monitora- 
mento. Por fim, a criação de uma rede de grupos de trabalho para a humanização dos serviços auxiliou na incorporação de profissionais das equipes na condução do projeto.

Os obstáculos foram, sobretudo, relacionados à redução da governabilidade da gestão municipal. A pequena autonomia do secretário municipal em gerir os recursos financeiros resultou em uma instabilidade no abastecimento da rede com suprimentos básicos (material de consumo e medicamentos) ao lado da precariedade do contrato dos médicos e das equipes do PSF. Esses obstáculos dificultaram a consolidação e a institucionalização de suas práticas e procedimentos na gestão municipal. A elevada adesão ao projeto revelou que o mesmo vinha ao encontro de um desejo dos profissionais nos diversos níveis. A naturalização das filas, atribuídas inicialmente por alguns profissionais e gerentes a uma suposta característica cultural da população, bem como o argumento da inevitabilidade de sua existência diante da baixa oferta revelaram-se falácias diante da efetividade das medidas adotadas, que não só foram capazes de extinguir as filas, dignificando a espera, como reduziram o tempo para a marcação da consulta. Além disso, o projeto mobilizou gerentes e profissionais que desenvolveram iniciativas criativas voltadas para a melhoria da qualidade do cuidado prestado. A presente experiência vem somar-se a diversas outras 10,20 que revelam a possibilidade de superação de barreiras organizacionais ao acesso aos serviços de saúde. Além disso, o discurso sobre a humanização do cuidado, no que toca às relações interpessoais, pode também passar da teoria à prática, quando algumas

\section{Referências}

1. Kopach R, De Laurentis P-C, Lawley M, Muthuraman K, Ozsen L, Rardin R, Wan H, Intrevado P, Qu X, Willis D. Effects of clinical characteristics on successful open access scheduling. Health Care Manag Sci. 2007; 10: 111-24.

2. Mallard SD, Leakeas T, Duncan WJ, Fleenor ME, Sinsky RJ. Same-day scheduling in a public health clinic: a pilot study. J Public Health Manag Pract. 2004; 10: 148-55.

3. Murray M, Berwick DM. Advanced Access: reducing waiting and delays in primary care. JAMA. 2003; 289 1035-40.

4. Knight AW, Padgett J, George B, Datoo MR. Reduced waiting times for GP: two exemples of "advanced access' in Australia. Med J Aust. 2005; 183: 101-3.

5. Travassos C, Oliveira EXG, Viacava F. Desigualdades geográficas e sociais no acesso aos serviços de saúde no Brasil: 1998 e 2003. Ciênc Saúde Coletiva. 2006; 11: $975-$ 86. estratégias de intervenção são implementadas em determinadas condições de possibilidade, como aquelas relatadas na presente experiência da gestão descentralizada do Sistema Único de Saúde.

Por fim, cabe refletir que a metodologia aqui utilizada para a avaliação da intervenção permitiu o acompanhamento da implantação das ações, a identificação dos progressos e obstáculos. Seus limites relacionam-se à complexa logística envolvendo recursos humanos que usualmente não estão disponíveis na rede básica de atenção à saúde. Por outro lado, o fato de ter havido incorporação de parte dos seus instrumentos e técnicas à gestão dos centros de saúde e equipes do PSF revela seu potencial como estratégia voltada para a institucionalização da avaliação. Em se tratando de uma ferramenta voltada aos processos de gestão e organização dos serviços, poderá ser aperfeiçoada e adaptada a outros contextos locais.

\section{Agradecimentos}

Agradecemos a Gleide Góes e Elide Azevedo, que fizeram parte da equipe de coordenação do projeto $\mathrm{e}$ supervisão das unidades, tendo participado ativamente em todas as fases do projeto; a Carlos Alberto Trindade, que participou da condução da primeira fase como coordenador da atenção básica e da segunda como secretário municipal de saúde; ao professor Jairnilson Paim, que participou das discussões sobre a concepção do projeto bem como do seu monitoramento; aos alunos da disciplina Políticas de Saúde, que participaram da análise da situação inicial.

6. IBGE (Instituto Brasileiro de Geografia e Estatística). Pesquisa Nacional por amostra de domicílios. Acesso e utilização de serviços de saúde: 2003. Rio de Janeiro; 2005.

7. Pinell P. Naissance d'un fléau. Histoire de la lutte contre le cancer en France (1890-1940). Paris: Éditions Métailié; 1992

8. Arouca ASS. O dilema preventivista. Contribuição para compreensão e crítica da medicina preventiva [tese]. Campinas: Faculdade de Ciências Médicas da Universidade Estadual de Campinas; 1975.

9. Brasil. Política Nacional de Humanização. Humaniza-SUS. Documento base para trabalhadores e gestores do SUS. Brasília, DF: Ministério da Saúde, Secretaria Executiva, Núcleo Técnico da Política Nacional de Humanização; 2004 
10. Deslandes SF. Análise do discurso oficial sobre humanização da assistência hospitalar. Ciênc Saúde Coletiva. 2004; 9: 7-13.

11. Puccini PT, Cecílio LCO. A humanização dos serviços e o direito à saúde. Cad Saúde Pública. 2004; 20: 1342-53.

12. Benevides R, Passos E. Humanização na saúde: réplica ao debate. Interface Comun Saúde Educ. 2005; 9: 389-406.

13. Deslandes SF. Humanização: revisitando o conceito a partir das contribuições da sociologia médica. In: Deslandes SF, org. Humanização dos cuidados em saúde: conceitos, dilemas e práticas. Rio de Janeiro: Fiocruz; 2006. página

14. Almeida $C$, organizadora. Os atalhos da mudança na saúde no Brasil: 9 estudos de caso. Rio de Janeiro: OPAS/OMS 1989. p. 19-27.

15. Paim JS. Modelos de atenção à saúde no Brasil. In Giovanella L, Escorel S, Lobato LVC, Noronha JC, Carvalho AI, orgs. Políticas e sistema de saúde no Brasil. Rio de Janeiro: Fiocruz; 2008. p. 547-7.

16. Pontes da Silva GA, Vieira-da-Silva LM. Health surveillance: proposal for a tool to evaluate technological arrangements in local health systems. Cad Saúde Pública. 2008; 24 2463-75.

17. Schraiber LB, Nemes MIB, Mendes-Gonçalves RB, organizadores. Saúde do adulto: programas e ações na unidade básica. São Paulo: Hucitec; 1996

18. São Paulo. Secretaria Municipal de Saúde. Acolhimento, o pensar, o fazer, o viver. São Paulo; 2002.

19. Solla JJSP. Acolhimento no sistema municipal de saúde. Rev Bras Saúde Matern Infant. 2005; 5: 493-503.

20. Franco TB, Bueno WS, Merhy EE. O acolhimento e os processos de trabalho em saúde: o caso de Betim, Minas Gerais, Brasil. Cad Saúde Pública. 1999; 15: 345-53.

21. Pichon-Rivière E. O processo grupal. São Paulo: Martins Fontes; 1988

22. Salvador. Secretaria Municipal de Saúde. Relatório da Assessoria Técnica da Secretaria Municipal de Saúde -

Recebido em 6 de maio de 2010

Versão final apresentada em 27 de setembro de 2010

Aprovado em 30 de setembro de 2010
TABWIN 2004-2006. Salvador; 2006

23. Brasil. Ministério da Saúde. Secretaria de Atenção à Saúde. Departamento de Atenção Básica. Evolução do credenciamento e implantação da estratégia Saúde da Família. Brasília, DF; 2007. [acessado 2007 nov]. Disponível em: http://dtr2004.saude.gov.br/dab/localiza/localiza_cadastro_ ret.php.

24. Pacientes passam noite na fila. $12^{\circ}$ Centro de Saúde, na Boca do Rio, atrai pessoas de vários bairros que chegam a esperar 24 horas. A Tarde 2005 Nov 29.

25. Salvador. Acolhimento na atenção básica em Salvador: plano para melhoria da acessibilidade. Salvador: Cooperação Técnica Instituto de Saúde Coletiva/ Secretaria de Saúde do Município de Salvador; 2005.

26. Salvador. Relatórios técnicos do plano de acolhimento e melhoria da acessibilidade 2005-2008. Salvador: Secretaria Municipal de Saúde de Salvador. Coordenação de Atenção e Prevenção à Saúde/ Instituto de Saúde Coletiva; 2008.

27. Vieira-da-Silva LM. Avaliação do processo de descentralização das ações de saúde. Ciênc Saúde Coletiva. 1999; 4: 331-9.

28. Vieira-da-Silva LM. Conceitos, abordagens e estratégias para a avaliação em saúde. In: Vieira-da-Silva LM, org. Avaliação em saúde: dos modelos teóricos à prática na avaliação de programas e sistemas de saúde. Salvador/Rio de Janeiro: EDUFBA/FIOCRUZ; 2005. p. 15-39.

29. Vieira-da-Silva LM, Lima Chaves SC, Esperidião MA, Lopes-Martinho RM. Accessibility to primary healthcare in the capital city of a northeastern state of Brazil: an evaluation of the results of a programme. J Epidemiol Comm Health. 2010. Published Online First 18 August 2010. doi:10.1136/jech.2009.097220

30. Souza LEPF. O SUS necessário e o SUS possível: gestão. Uma reflexão a partir de uma experiência concreta. Ciênc Saúde Coletiva. 2009; 14: 911-8. 